\title{
Link between IL-10 and activation of the NLRP3 inflammasome in the synovium
}

New research in mouse models of inflammatory arthritis shows that IL-10 is a negative regulator of NLRP3 inflammasome components within the inflamed synovium. "Our studies implicate a link between IL-10 and expression of the NLRP3 inflammasome within the inflamed synovium, and advocate a role for IL-10 and inflammasome activation in governing osteoclastogenesis and bone erosion," comments Professor Simon Jones, corresponding author of the study now published in Arthritis Research \& Therapy.

Activation of the NLRP3 inflammasome has been implicated in various autoimmune and autoinflammatory conditions, but little is known about the regulation of inflammasome components in inflammatory arthritis. IL-10 is an antiinflammatory cytokine that modulates both innate and cellular immunity through inhibition of signalling via a wide range of pattern recognition receptors, including NLRs. To investigate whether IL-10 is
44 ...the changes in IL-1 $\beta$ expression reflect the capacity of IL-10 to inhibit expression of II1b, NIrp3... 77

involved in regulation of inflammasome activation in the synovium, the researchers studied antigen-induced arthritis (AIA) in IL-10-knockout and wild-type mice.

Greenhill et al. showed that IL-10knockout mice in the late stages of AIA had an exacerbation of joint pathology in comparison with wildtype mice, and this was associated with increased synovial expression of NLRP3 inflammasome components and increased expression of IL- $1 \beta$ at bone erosion sites, which co-localized with F4/80+ resident macrophages.

They propose that the changes in IL- $1 \beta$ expression reflect the capacity of IL-10 to inhibit the expression of Illb, Nlrp3 and genes encoding other inflammasome components. Thus, targeting the inflammasome might be beneficial in diseases with increased IL- $1 \beta$ production, such as osteoarthritis, Muckle-Wells syndrome, and gout. "The use of smallmolecule inhibitors that block NLRP3 activity may have downstream therapeutic potential," notes Jones, "and we now need to consider whether this is relevant to human disease."

"Monitoring IL-10 or inflammasome activities within the inflamed joints of patients with early rheumatoid arthritis may prove valuable as a predictor of disease activity or bone erosions," states Jones, "and may help tailor the design of novel treatments for defined patient groups."

Caroline Barranco

Original article Greenhill, C. et al. Interleukin-10 regulates the inflammasome-driven augmentation of inflammatory arthritis and joint destruction. Arthritis Res. Ther. doi:10.1186/s13075-014-1419-y 